\title{
Fast, non-diffusive ignition of a gaseous reacting mixture subject to a point energy source
}

\author{
C Vázquez-Espí
}

\begin{abstract}
A Liñán
The ignition of a gaseous reactive mixture subject to a localized energy source is analysed using large activation energy asymptotics. The energy released by the source results in a thermal non-uniformity in a small region of the gas. We distinguish two different regimes, non-diffusive and diffusive, depending on the dominant cooling mechanism during the ignition stage: expansion effects or heat conduction. We focus on the non-diffusive ignition, considering the energy source as instantaneous. We show the existence of a critical value of a Damköhler number, defined as the ratio of the characteristic times of the expansion waves and chemical reaction, such that ignition only occurs for supercritical values at a well-defined ignition time, which is calculated numerically. The ignition process for a non-instantaneous energy source is also described in terms of an initial inert heating stage and a shorter reactive stage ending in thermal runaway for supercritical values of the Damköhler number.
\end{abstract}

\section{Introduction}

The global response of the reactions in combustion is strongly dependent on temperature. Since at the ambient conditions the chemical reaction typically proceeds at such a very low rate that the cooling mechanisms are able to prevent the temperature in the reactive mixture from rising significantly, the initiation of a combustion wave in a cold reactive gas requires an external stimulus to increase the reaction rate locally. One common way is to create a thermal non-uniformity, or hot-spot, by the deposition of a certain amount of energy in a small region; the resulting increase in temperature can lead to an ignition event, or local thermal runaway, at a well-defined time, and, after ignition, the process may eventually evolve into either a deflagration or a detonation wave.

Whether ignition occurs or not is the result of the competition between two opposing effects. On one hand, the capability of the thermal non-uniformity for triggering the chemical reaction, which can be measured in terms of the homogeneous ignition time as a function of 
the peak temperature generated by the external source, and, on the other hand, the cooling mechanisms that tend to smooth the non-uniformity. In the absence of radiative transfer these are related to the effects of heat conduction transport or the expansion waves associated with the compressibility of the gas. Depending on the dominant mechanism, we can distinguish two ignition regimes: a slow diffusive regime and a fast non-diffusive one. Kassoy et al [1] have addressed a systematic discussion of the equations governing the different subregimes that appear for both diffusive and non-diffusive ignition. In the diffusive regime they show the existence of a critical value of a Damköhler number for thermal runaway to occur. However, they do not consider the equivalent problem in the non-diffusive regime, in which there also exists a critical value of an appropriate Damköhler number for non-diffusive ignition to occur.

Our aim is to obtain the critical conditions leading to short-time ignition at a hot-spot for a mixture in which the chemical reaction can be modelled by an overall Arrhenius reaction with large activation energy, although the analysis should be generalized to more realistic kinetic schemes. We can expect to find an ignition event characterized by a thermal runaway occurring first at the point of maximum reaction rate, say at the centre of the hot-spot where the temperature attains its maximum value $T_{\mathrm{m}}$. For a one-step, irreversible, Arrhenius reaction with activation energy $E$ much larger than the thermal energy $R T$, the Frank-Kamenetskii temperature $R T_{\mathrm{m}}^{2} / E$ defines the order of magnitude of the increase, or decrease, in temperature required to change the reaction time by a factor of order unity. The critical conditions will be determined by the competition, in the core region of the hot-spot where $T$ differs from $T_{\mathrm{m}}$ by an amount $R T_{\mathrm{m}}^{2} / E$, between the heating due to the reaction and cooling by either the expansion waves or the heat conduction. The result depends on the relative values of the corresponding characteristic times, $t_{\mathrm{ch}}, t_{\mathrm{a}}$ and $t_{\mathrm{c}}$, required to change the temperature in the core of the hot-spot by an amount of order $R T_{\mathrm{m}}^{2} / E$. The first, $t_{\mathrm{ch}}$, is the homogeneous ignition time at temperature $T_{\mathrm{m}}$; the second, $t_{\mathrm{a}}$, is the time required by acoustic waves to move across the core of the hot-spot; and the third, $t_{\mathrm{c}}$, is the corresponding time of heat conduction. It is important to note that the ratio $t_{\mathrm{a}} / t_{\mathrm{c}}$ is also the Knudsen number, or ratio of the meanfree-path to the size of the core of the hot-spot; this is typically very small compared with unity, and should be so for the continuum description to be applicable. Therefore, in the following we consider that $t_{\mathrm{a}} \ll t_{\mathrm{c}}$, and it follows that the ignition process is diffusive if $t_{\mathrm{a}} \ll t_{\mathrm{ch}} \lesssim t_{\mathrm{c}}$, or non-diffusive if $t_{\mathrm{ch}} \lesssim t_{\mathrm{a}} \ll t_{\mathrm{c}}$. The limit of Knudsen numbers of order unity has been considered by Clarke et al [2], who describe the evolution to a detonation of a reacting gas mixture when the energy addition takes place in very short scales in time and space.

Let us start by assuming that the time of external heat addition is much smaller than the other characteristic times. If, after the external energy addition, the conditions are such that $t_{\mathrm{ch}} \ll t_{\mathrm{c}}$, the effects of the diffusive transport mechanisms are negligible at times of order $t_{\mathrm{ch}}$ and we find the non-diffusive regime. As pointed out by Kassoy et al [1], there is a distinguished regime, for $t_{\mathrm{ch}} \sim t_{\mathrm{a}}$, in which the heating due to the chemical reaction has to compete with the cooling due to the expansion. Jackson et al [3] have analysed this limit in a confined system in which the temperature disturbances, of the order of the Frank-Kamenetskii temperature, are not localized in a small region but extend to the whole system. Since they assumed that the initial pressure is uniform and the gas is motionless, the system, for times much smaller than the conduction time, does not have any mechanism to smooth the thermal non-uniformity and to reduce the effect of the reaction heating. Consequently, they find a thermal runaway for any container size.

Zel'dovich et al [4] have treated numerically the evolution of a non-uniformly heated gas. This problem was revisited by Kapila and Dold [5], who provided an analytical description. The initial temperature profile is assumed to be linear and its non-dimensional gradient is the 
parameter that determines the evolution of the system. As in [3], due to the uniform initial pressure, there is no cooling by expansion waves and a thermal runaway occurs for all values of this parameter, which only determines whether the subsequent evolution ends in a detonation or deflagration. Short [6] has considered, for $t_{\mathrm{a}}=t_{\mathrm{ch}}$, the evolution of a reactive gaseous mixture with small initial disturbances in velocity and pressure gradient, both linear in space. He finds that the velocity, pressure and temperature evolve homogeneously and obtains the range of values of the parameters characterizing the initial disturbances that lead to ignition.

In the limiting cases with $t_{\mathrm{ch}} / t_{\mathrm{a}} \ll 1$, ignition occurs without significant expansion effects. The corresponding constant volume explosion is described by Kassoy et al [1]. In the opposite limiting cases, when $t_{\mathrm{ch}} / t_{\mathrm{a}} \gg 1$, the overpressures generated by the external heat release disappear, in unconfined systems, at times of order $t_{\mathrm{a}}$, leaving a heated spot with a significantly reduced peak temperature. The corresponding reaction time increases strongly from $t_{\mathrm{ch}}$ to a much larger value $t_{\mathrm{ch}}^{\prime}$, which must be smaller than the heat conduction time to ensure a thermal runaway. In confined systems, with hot-spots of the size of the system, analysed by Kassoy et al [7], the reduction in peak temperature is not so large and the increase in ignition time is not strong.

The diffusive, or slow, ignition occurs when $t_{\mathrm{c}} \sim t_{\mathrm{ch}}^{\prime}$. We find ignition if the ratio $t_{\mathrm{c}} / t_{\mathrm{ch}}^{\prime}$, which is the classical Damköhler number for the perturbed region, is greater than a critical value, i.e. if the conductive heat losses from the hot-spot to the surroundings are overcome by the heat released by the chemical reaction. This case has been treated by Berman et al [8] and we revisit it in [9], where we consider the flame initiation problem.

In this paper, we analyse the evolution of a uniform gaseous reacting mixture when subject to a localized deposition of energy in a region of the system with a volume small compared to the volume of the chamber, so that the system can be considered as unconfined. We assume that the external energy addition takes place instantaneously, without changes in density. Therefore, we find a hot-spot with initial disturbances in both temperature and pressure. Moreover, we consider that the chemical time is very small compared to the conduction time $\left(t_{\mathrm{ch}} \ll t_{\mathrm{c}}\right)$, so that the evolution is influenced only by chemical reaction and expansion effects, whose characteristic times, $t_{\mathrm{ch}}$ and $t_{\mathrm{a}}$, are assumed to be of the same order. After the addition of energy, the gradients due to the overpressures generate expansion waves that smooth the non-uniformity. The heat released by the chemical reaction does or does not overpower the drop in temperature depending on the Damköhler number $t_{\mathrm{a}} / t_{\mathrm{ch}}$, which measures the relative importance of the two effects. As we show in section 2, there exists a critical value such that only for supercritical values an ignition event is found at an ignition time of order $t_{\mathrm{ch}}$, which can be identified by the typical logarithmic growth of both temperature and pressure with time.

In order to account for the non-zero duration of the external heat addition, we need to describe the simultaneous external and reactive heating. At early times, the fluid variables are given by their inert values, without the effects of the chemical reaction. These become significant only when the maximum temperature approaches a cross-over value, giving rise to a short reactive stage, described in section 3.1 , which may result in a thermal runaway. When the external heat addition is limited to a finite time, the inert temperature reaches a maximum value and then decreases; if this maximum temperature is lower than the cross-over temperature, a thermal runaway may not occur. The corresponding critical conditions for ignition to occur will be given in section 3.2.

\section{Non-diffusive, fast ignition}

Let us consider a gaseous reacting mixture, with uniform initial conditions $\rho_{0}, p_{0}$ and $T_{0}$, that is perturbed symmetrically by the addition of an amount of energy, $\dot{Q}(r, t)$ per unit volume 
and time, in a domain of characteristic length $r_{\mathrm{h}}$. We assume that the addition of energy takes place during an interval of duration $t_{\mathrm{e}}$, i.e. $\dot{Q}(r, t)=0$ for $t>t_{\mathrm{e}}$, which is small compared to the acoustic time $r_{\mathrm{h}} / \sqrt{\gamma R_{\mathrm{g}} T_{0}}$. Hence, we can consider that an instantaneous energy source releases a density of energy $Q(r)=\int_{0}^{t_{\mathrm{e}}} \dot{Q}(r, t) \mathrm{d} t$. As a result of this external energy addition, we generate a hot-spot in the reacting gas, and the temperature $T$ and the pressure $p$ jump from their initial values, $T_{0}$ and $p_{0}$, to the new values $T_{\mathrm{h}}$ and $p_{\mathrm{h}}$ given, at $t=0^{+}$, by

$$
T_{\mathrm{h}}(r) / T_{0}=p_{\mathrm{h}}(r) / p_{0}=1+Q(r) / \rho_{0} c_{\mathrm{v}} T_{0},
$$

while the density keeps its initial value $\rho_{0}$, because there is no time for the generation of motion in the gas during the external energy addition. We assume that $Q(r)$, symmetrical around $r=0$, is bell shaped and such that $T_{\mathrm{h}}(r)$ is significantly different from $T_{0}$ only for $r \sim r_{\mathrm{h}}$, with its maximum value $T_{\mathrm{m}}=T_{\mathrm{h}}(0)$.

For the times considered, which are small compared to the heat conduction time across the hot-spot, the evolution of the gaseous mixture is given by

$$
\begin{aligned}
& p=\rho R_{\mathrm{g}} T, \\
& \frac{\partial \rho}{\partial t}=-\frac{1}{r^{j}} \frac{\partial}{\partial r}\left(r^{j} \rho u\right), \\
& \frac{\mathrm{D} u}{\mathrm{D} t}=-\frac{1}{\rho} \frac{\partial p}{\partial r}, \\
& \rho c_{\mathrm{p}} \frac{\mathrm{D} T}{\mathrm{D} t}=\frac{\mathrm{D} p}{\mathrm{D} t}+\rho q Y_{0} Y B \mathrm{e}^{-E / R T}, \\
& \rho \frac{\mathrm{D} Y}{\mathrm{D} t}=-\rho Y B \mathrm{e}^{-E / R T},
\end{aligned}
$$

where $\mathrm{D} / \mathrm{D} t=\partial / \partial t+u \partial / \partial r$ and $q$ is the heat of reaction per unit mass of reactant. These equations correspond to the equation of state and the conservation of mass, momentum, energy and mass of fuel, whose mass fraction, $Y$, has been scaled with its initial value $Y_{0}$. Equations (2)-(6) are to be solved, for $t>0$ and $r>0$, with the initial conditions

$$
t=0: \quad u=\rho-\rho_{0}=T-T_{\mathrm{h}}(r)=p-p_{\mathrm{h}}(r)=Y-1=0,
$$

and symmetry conditions at $r=0$,

$$
r=0: \quad u=\frac{\partial \rho}{\partial r}=\frac{\partial T}{\partial r}=\frac{\partial p}{\partial r}=\frac{\partial Y}{\partial r}=0 .
$$

For $r \rightarrow \infty$ the variables are given by

$$
r \rightarrow \infty: \quad u=\rho-\rho_{0}=T-T_{\infty}=p-p_{\infty}=Y-Y_{\infty}=0,
$$

where $T_{\infty}(t), p_{\infty}(t)$ and $Y_{\infty}(t)$ are the solutions of the system of equations

$$
\begin{aligned}
& \frac{\mathrm{d} T_{\infty}}{\mathrm{d} t}=\frac{q Y_{0}}{c_{v}} Y_{\infty} B \mathrm{e}^{-E / R T_{\infty}}, \\
& Y_{\infty}=1-\frac{c_{v}}{q Y_{0}}\left(T_{\infty}-T_{0}\right), \\
& p_{\infty}=\rho_{0} T_{\infty} R_{\mathrm{g}},
\end{aligned}
$$

corresponding to the homogeneous, constant-density form of (2)-(6), with the initial conditions $T_{\infty}(0)=T_{0}, Y_{\infty}(0)=1$ and $p_{\infty}(0)=p_{0}$. The well-known solution of (10) and (11), for $E / R T_{\infty} \gg 1$, shows that, in a first ignition stage with a characteristic time $t_{\infty}$ defined by $t_{\infty}=\left(R T_{0} / E\right)\left(c_{\mathrm{v}} T_{0} / q Y_{0}\right) B^{-1} \mathrm{e}^{E / R T_{0}}$, the Arrhenius exponent can be linearized around $T_{0}$ and the reactant consumption can be neglected if $\left(c_{\mathrm{v}} T_{0} / q Y_{0}\right)\left(R T_{0} / E\right) \ll 1$. Then the solution of 
(10) is given by $T_{\infty}-T_{0}=-\left(R T_{0}^{2} / E\right) \ln \left(1-t / t_{\infty}\right)$, showing blow-up at the self-ignition time $t_{\infty}$.

However, due to the external heat release and the strong sensitivity of the chemical reaction to temperature, we can expect to encounter significant chemical effects for $t \ll t_{\infty}$ around the centre of the hot-spot, $r=0$, where $T=T_{\mathrm{m}}>T_{0}$ at $t=0^{+}$. Without expansion and transport effects, the chemical reaction would lead to a thermal runaway at a time $t_{\mathrm{R}}$ defined by $t_{\mathrm{R}}=\left(R T_{\mathrm{h}} / E\right)\left(c_{\mathrm{v}} T_{\mathrm{h}} / q Y_{0}\right) B^{-1} \mathrm{e}^{E / R T_{\mathrm{h}}}$. Its minimum value is $t_{\mathrm{ch}}=t_{\mathrm{R}}(0)$, given by

$$
t_{\mathrm{ch}}=\frac{R T_{\mathrm{m}}}{E} \frac{c_{\mathrm{v}} T_{\mathrm{m}}}{q Y_{0}} B^{-1} \mathrm{e}^{E / R T_{\mathrm{m}}},
$$

which is exponentially large compared to $t_{\infty}$ if the Zel'dovich number, $E\left(T_{\mathrm{m}}-T_{0}\right) / R T_{\mathrm{m}}^{2}$, is large compared to unity. The local ignition time $t_{\mathrm{R}}$ will be of order $t_{\mathrm{ch}}$ only in the FrankKamenetskii region, at the core of the hot-spot, where $T_{\mathrm{h}}(r)-T_{\mathrm{m}} \sim R T_{\mathrm{m}}^{2} / E \ll T_{\mathrm{m}}$ if $\varepsilon=R T_{\mathrm{m}} / E \ll 1$. At times of order $t_{\mathrm{ch}}$, the chemical reaction will produce changes of order $R T_{\mathrm{m}}^{2} / E$ in the temperature only in the Frank-Kamenetskii region. Elsewhere, the reaction appears to be frozen.

On the other hand, in the non-reactive limit, $B=0$, for times small compared to the acoustic time through the hot-spot $r_{\mathrm{h}} / c_{\mathrm{m}}$, where $c_{\mathrm{m}}=\sqrt{\gamma R_{\mathrm{g}} T_{\mathrm{m}}}$ is the velocity of sound at the initial peak temperature, the changes in $u, \rho, T$ and $p$ due to the expansion are given by the solution of (2)-(6) with $B=0$, leading to

$$
\begin{aligned}
& u=-t R_{\mathrm{g}} \frac{\mathrm{d} T_{\mathrm{h}}}{\mathrm{d} r}+\mathrm{O}\left(t^{3}\right), \\
& \frac{\rho-\rho_{0}}{\rho_{0}}=\frac{1}{2} R_{\mathrm{g}} t^{2} \Delta T_{\mathrm{h}}+\mathrm{O}\left(t^{4}\right), \\
& \frac{T-T_{\mathrm{h}}}{T_{\mathrm{h}}}=\frac{1}{2} R_{\mathrm{g}} t^{2}\left((\gamma-1) \Delta T_{\mathrm{h}}+\frac{1}{T_{\mathrm{h}}}\left(\frac{\mathrm{d} T_{\mathrm{h}}}{\mathrm{d} r}\right)^{2}\right)+\mathrm{O}\left(t^{4}\right), \\
& \frac{p-p_{\mathrm{h}}}{p_{\mathrm{h}}}=\frac{1}{2} R_{\mathrm{g}} t^{2}\left(\gamma \Delta T_{\mathrm{h}}+\frac{1}{T_{\mathrm{h}}}\left(\frac{\mathrm{d} T_{\mathrm{h}}}{\mathrm{d} r}\right)^{2}\right)+\mathrm{O}\left(t^{4}\right),
\end{aligned}
$$

where $\Delta$ is the symmetric form, $r^{-j}(\mathrm{~d} / \mathrm{d} r)\left(r^{j} \mathrm{~d} / \mathrm{d} r\right)$, of the Laplacian operator.

If the hot-spot produced by the external energy release is not flat at the centre, we can define $r_{\mathrm{h}}$ in terms of the curvature of the initial profile as $r_{\mathrm{h}}^{2}=-2 T_{\mathrm{m}} /\left(\mathrm{d}^{2} T_{\mathrm{h}} / \mathrm{d} r^{2}\right)_{r=0}$. The characteristic size of the Frank-Kamenetskii region is then defined by $r_{\mathrm{c}}=\sqrt{\varepsilon} r_{\mathrm{h}}$, and the initial temperature for $r \sim r_{\mathrm{c}}$ can be represented by $T_{\mathrm{h}}=T_{\mathrm{m}}\left(1-\varepsilon\left(r / r_{\mathrm{c}}\right)^{2}\right)$. Thus, without the effects of the chemical reaction, the temperature in the core region of the hot-spot is given, for $t \ll r_{\mathrm{h}} / c_{\mathrm{m}}$, by the relation

$$
\frac{T-T_{\mathrm{m}}}{\varepsilon T_{\mathrm{m}}}=-\left(\frac{r}{r_{\mathrm{c}}}\right)^{2}-\frac{\gamma-1}{\gamma}(j+1)\left(\frac{t}{t_{\mathrm{a}}}\right)^{2},
$$

where $t_{\mathrm{a}}=r_{\mathrm{c}} / c_{\mathrm{m}}$ is the acoustic time through the Frank-Kamenetskii region. Notice that the non-reactive solution would not have exhibited this cooling mechanism if the energy deposition had taken place at constant pressure, giving rise to initial conditions analogous to those of $[3,5]$.

Therefore, we find that the pressure expansion waves lead, according to (18), to a drop in temperature of order $\varepsilon T_{\mathrm{m}}$ at times of the order of the acoustic time $t_{\mathrm{a}}$, while the chemical reaction, with the peak initial reaction rate, requires times of order $t_{\mathrm{ch}}$ to increase $T$ by the same amount. Whether ignition occurs at times of order $t_{\mathrm{a}}$ or not depends on the ratio $t_{\mathrm{a}} / t_{\mathrm{ch}}$. If $t_{\mathrm{ch}}$ is large compared to $t_{\mathrm{a}}$, the expansion decreases the temperature and, hence, the reaction rate. The time required for the appearance of significant thermal effects due to the chemical 
reaction becomes much larger that $t_{\mathrm{ch}}$, and we do not find ignition event at times of order $t_{\mathrm{a}}$. If, on the other hand, $t_{\mathrm{ch}}$ is shorter than $t_{\mathrm{a}}$, there is no time to transform thermal energy into kinetic energy and the ignition occurs at $t=t_{\mathrm{ch}}$, without changes in density. For $t_{\mathrm{a}} \sim t_{\mathrm{ch}}$, there is a competition between expansion cooling and reactive heating and we find an ignition event for values of the ratio $t_{\mathrm{a}} / t_{\mathrm{ch}}$ larger than a critical value to be determined.

We now describe the evolution of the reacting gas in the core region of the hot-spot, $r \sim r_{\mathrm{c}}$, at times of the order of the acoustic time $t_{\mathrm{a}}$ when $t_{\mathrm{ch}}$, defined by (13), is also of order $t_{\mathrm{a}}$. An order-of-magnitude estimate of the changes in the fluid dynamic variables, based on the early-time description, (14)-(17), of the chemically frozen expansion wave for $r \sim r_{\mathrm{c}}$ and $t \sim t_{\mathrm{a}}$, shows that it is convenient to use

$$
\begin{aligned}
& \tilde{u}=u / \varepsilon c_{\mathrm{m}}, \quad \tilde{\rho}=\left(\rho-\rho_{0}\right) / \varepsilon \rho_{0}, \\
& \tilde{T}=\left(T-T_{\mathrm{m}}\right) / \varepsilon T_{\mathrm{m}}, \quad \tilde{p}=\left(p-p_{\mathrm{m}}\right) / \varepsilon p_{\mathrm{m}},
\end{aligned}
$$

as dependent variables which are expected to be of order unity for values of the independent variables $\xi=r / r_{\mathrm{c}}$ and $\tau=t / t_{\mathrm{a}}$, also of order unity in the core region of the hot-spot at times of the order of the acoustic time.

When equations (2)-(6) are rewritten in terms of the new variables, we find that for $\varepsilon \ll 1$ and $\xi$ and $\tau$ of order unity, during the ignition stage, the equations, at leading order in $\varepsilon$, simplify to $Y=1$ and

$$
\begin{aligned}
& \tilde{p}=\tilde{\rho}+\tilde{T}, \\
& \frac{\partial \tilde{\rho}}{\partial \tau}=-\frac{1}{\xi^{j}} \frac{\partial\left(\xi^{j} \tilde{u}\right)}{\partial \xi}, \\
& \frac{\partial \tilde{u}}{\partial \tau}=-\frac{1}{\gamma} \frac{\partial \tilde{p}}{\partial \xi}, \\
& \frac{\partial \tilde{T}}{\partial \tau}=\frac{\gamma-1}{\gamma} \frac{\partial \tilde{p}}{\partial \tau}+\delta \mathrm{e}^{\tilde{T}},
\end{aligned}
$$

to be solved for $\tau>0$, with the initial conditions

$$
\tau=0: \quad \tilde{u}=\tilde{\rho}=\tilde{T}+\xi^{2}=\tilde{p}+\xi^{2}=0,
$$

and symmetry conditions at $\xi=0$. For large values of $\xi$, not yet reached at $\tau \sim 1$ by the effects of the chemical reaction, which are important only for $\xi \sim 1$, the fluid variables take their chemically frozen values. From (14)-(17) it follows that

$\xi \gg 1: \quad \tilde{u}=2 \xi \tau / \gamma, \quad \gamma \tilde{\rho}=\gamma\left(\tilde{T}+\xi^{2}\right) /(\gamma-1)=\tilde{p}+\xi^{2}=-(j+1) \tau^{2}$,

which is the exact solution of (20)-(23) with $\delta=0$. The only parameters remaining in this system of equations are the specific-heat ratio, $\gamma=c_{\mathrm{p}} / c_{\mathrm{v}}$, and the Damköhler number, $\delta=t_{\mathrm{a}} / \gamma t_{\mathrm{ch}}$. Notice that, except for the non-linear effects appearing in the reaction term, these equations are the linearized form of (2)-(6) in the core of the hot-spot, and lead to Clarke's equation $\left(\tilde{T}_{\tau}-\gamma \delta \mathrm{e}^{\tilde{T}}\right)_{\tau \tau}=\xi^{-j}\left(\xi^{j}\left(\tilde{T}_{\tau}-\delta \mathrm{e}^{\tilde{T}}\right)_{\xi}\right)_{\xi}$.

We may expect that the problem (20)-(24) will exhibit a thermal runaway or blow-up, i.e. $\tilde{T}(0, \tau) \rightarrow \infty$ at $\tau=\tau_{\mathrm{i}}(\delta, \gamma) \sim 1$, for values of the Damköhler number larger than a critical value $\delta_{\mathrm{c}}(\gamma)$; while for values $\delta<\delta_{\mathrm{c}}$ ignition will not occur.

\subsection{Numerical results}

Before solving numerically the equations (20)-(23) it is convenient to eliminate the density, by means of the equation of state, and consider as dependent variables the increments in temperature, pressure and velocity due to the chemical reaction, $\varphi, \pi$ and $v$, respectively, 
which are defined as the difference of $\tilde{T}, \tilde{p}$ and $\tilde{u}$ with the corresponding inert solution given by (25). Then the problem to be solved can be written as

$$
\begin{aligned}
& \frac{\partial v}{\partial \tau}+\frac{1}{\gamma} \frac{\partial \pi}{\partial \xi}=0, \\
& \frac{\partial \varphi}{\partial \tau}-\frac{\gamma-1}{\gamma} \frac{\partial \pi}{\partial \tau}=\delta \exp \left(\varphi-\frac{\gamma-1}{\gamma}(1+j) \tau^{2}-\xi^{2}\right), \\
& \frac{1}{\gamma} \frac{\partial \pi}{\partial \tau}+\frac{1}{\xi^{j}} \frac{\partial\left(\xi^{j} v\right)}{\partial \xi}=\delta \exp \left(\varphi-\frac{\gamma-1}{\gamma}(1+j) \tau^{2}-\xi^{2}\right),
\end{aligned}
$$

with symmetry conditions at $\xi=0$ and homogeneous initial conditions. These equations are to be solved by a marching-on-time procedure, starting with the solution of (26)-(28) for small values of $\tau$. This is given by $\varphi=\tau \varphi_{1}(\xi)+\tau^{2} \varphi_{2}(\xi)+\cdots, \pi=\tau \pi_{1}(\xi)+\tau^{2} \pi_{2}(\xi)+\cdots$ and $v=\tau^{2} v_{2}(\xi)+\cdots$, where $\varphi_{1}=\pi_{1}=\gamma \delta \mathrm{e}^{-\xi^{2}}, \varphi_{2}=\pi_{2}=\varphi_{1}^{2} / 2$ and $v_{2}=\delta \xi \mathrm{e}^{-\xi^{2}}$.

This system of equations is suitable to be integrated along the characteristics. However, in the cylindrical and spherical cases $(j \neq 0)$ the equivalent system of ordinary differential equations becomes weakly unstable. Instead, we have carried out the numerical integration of (26)-(28) by means of an implicit finite-difference scheme with a non-uniform mesh, the finest grid spacing located around $\xi=0$. We use a variable time-step in order to determine accurately the ignition time in the supercritical cases. After discretization, we obtain a system of non-linear algebraic equations involving the values of $\varphi, \pi$ and $v$ at each point of the mesh at a given value of $\tau$. Elementary algebraic manipulations allow us to write the system in uncoupled form, and we only need to solve a reduced system of non-linear equations for the vector of unknowns involving the values of $\varphi$.

Jackson et al [3] have described the evolution of the temperature at the core of the hot-spot during the final stage of the ignition period. Their calculations can be adapted to the present case with only minor changes. In particular, we find that the temperature increment, $\varphi$, at the centre of the hot-spot behaves as $-\ln \left(\gamma \delta\left(\tau_{\mathrm{i}}-\tau\right)\right)+(\gamma-1)(j+1) \tau_{\mathrm{i}}^{2} / \gamma+\mathrm{O}\left(\left(\tau_{\mathrm{i}}-\tau\right) \ln \left(\tau_{\mathrm{i}}-\tau\right)\right)$, which we use to compute accurately the value of $\tau_{\mathrm{i}}(\delta, \gamma)$. Figure 1 shows the temperature, $\varphi(0, \tau)$, and the pressure, $\pi(0, \tau)$, at the centre of the hot-spot as functions of $\tau$ for $\gamma=1.4$ and different values of $\delta$. We find that the critical values of the Damköhler number are 0.50340 , 0.73583 and 0.91448 for the planar, cylindrical and spherical cases, respectively. Figure 2 shows the ignition time as a function of $\delta$.

In the subcritical cases the chemical reaction becomes frozen at times $\tau \gg 1$, because of the decrease in the inert temperature. Most of released thermal energy in the reaction stage is converted into kinetic energy resulting in a pressure wave that propagates at the speed of sound. However, $\varphi(0, \tau)$ tends to a constant value as $\tau \rightarrow \infty$, such that $\varphi(0, \infty)$ grows when $\delta$ approaches $\delta_{\mathrm{c}}$ (see figure 1). This residual increment in temperature disappears only as a result of heat conduction, which requires a larger time scale.

When $\delta$ is supercritical, the maximum value of the increment in temperature due to the chemical reaction is monotonically increasing with time. The behaviour of the pressure is quite different. For large values of $\delta$, the increments in pressure and temperature show a similar growth. However, as $\delta$ is reduced towards $\delta_{\mathrm{c}}$, and the ignition time becomes large compared to the acoustic time, the overpressures generated by the peak reaction rate are overcome by the expansion waves, and ignition occurs after the pressure has reached a maximum value (see figure 1). Note also the effect of the geometry on the pressure increment at the centre of the hot-spot.

For very large values of $\delta$, ignition occurs before the pressure waves have time to change the density. The characteristic time is now $t_{\mathrm{ch}}$ and the appropriate time variable is $\sigma=\gamma \delta \tau$. The solution of (26)-(28) can be sought as an expansion in powers of $\delta^{-1}$, obtaining, for 


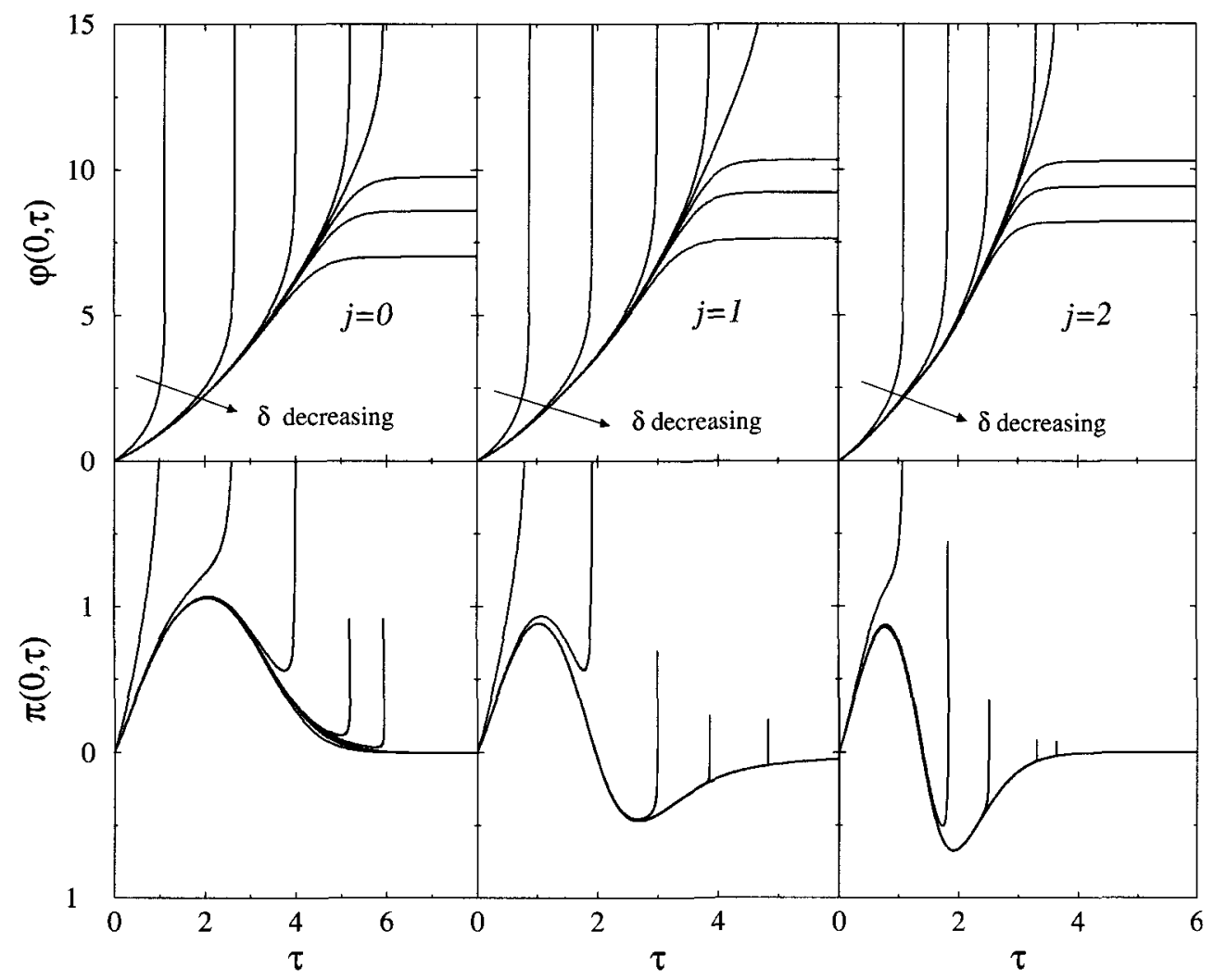

Figure 1. Temperature, $\varphi(0, \tau)$, and pressure, $\pi(0, \tau)$, at the centre of the hot-spot as a function of time $\tau$ for the following values of the Damköhler number (critical values are typeset in bold). $j=0: \delta=0.75,0.51563,0.50391,0.50342, \mathbf{0 . 5 0 3 4 0}, 0.50339,0.50336,0.50317 ; j=1: \delta=1$, $0.75,0.73633,0.73584, \mathbf{0 . 7 3 5 8 3}, 0.73581,0.73578,0.73560 ; j=2: \delta=1,0.92188,0.91504$, $0.91449,0.91448,0.91446,0.91443,0.91431$.

the leading term, $\varphi=\pi=-\ln \left(1-\sigma \mathrm{e}^{-\xi^{2}}\right)$, and $v=-2 \delta^{-1} \gamma^{-2} \xi\left(\sigma+\mathrm{e}^{\xi^{2}} \ln \left(1-\sigma \mathrm{e}^{-\xi^{2}}\right)\right)$. Therefore, the ignition time for $\delta \gg 1$ is, in first approximation, independent of the geometry and given by $\tau_{\mathrm{i}}(\delta \gg 1, \gamma)=(\gamma \delta)^{-1} \ll 1$, or $t_{\mathrm{i}}=t_{\mathrm{ch}}$ in dimensional variables, in agreement with the numerical results (see figure 2 ).

\section{Fast ignition with a non-instantaneous energy source}

In the previous section we have analysed the ignition process when the time of external heat addition is very short compared to the acoustic time. In the following, we describe the ignition process when the time of external heat release is of the order of the acoustic time. The large activation energy limit will allow us to distinguish two stages in the process: a first inert stage in which the chemical reaction is frozen and the fluid variables are determined by the external heating, and a very short reactive stage ending in the thermal runaway.

Starting at $t=0$, a source releases heat at a rate $\dot{Q}(r, t)$ in a region of characteristic size $r_{\mathrm{h}}$ during a time $t_{\mathrm{e}}$ that is of the same order as the acoustic time $r_{\mathrm{h}} / \sqrt{\gamma R_{\mathrm{g}} T_{0}}$. We assume that the function $\dot{Q}(r, t)$ is symmetrical around $r=0$ and bell shaped, so that $\dot{Q}(r, t)$ is significantly 


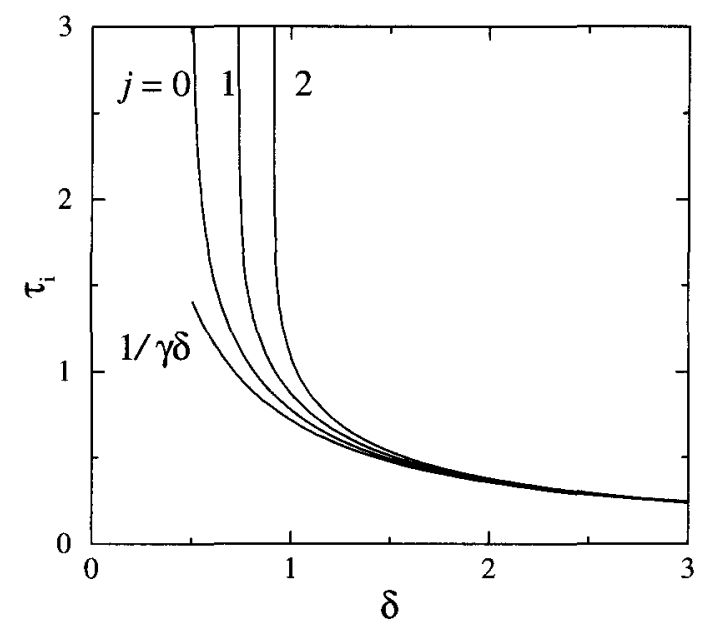

Figure 2. The ignition time $\tau_{\mathrm{i}}$ as a function of $\delta$ for $\gamma=1.4$.

different from zero only in $r<r_{\mathrm{h}}$. Moreover, $\dot{Q}(r, t)=0$ for $t>t_{\mathrm{e}}$ and

$$
\int_{0}^{t_{\mathrm{e}}} \int_{0}^{\infty} \dot{Q}(r, t) r^{j} \mathrm{~d} r \mathrm{~d} t \sim r_{\mathrm{h}}^{j+1} \rho_{0} c_{\mathrm{p}} T_{0} .
$$

The evolution of the gas mixture for times small compared to the conduction time is given by equations (2)-(6), with (5) replaced by

$$
\rho c_{\mathrm{p}} \frac{\mathrm{D} T}{\mathrm{D} t}=\frac{\mathrm{D} p}{\mathrm{D} t}+\rho q Y_{0} Y B \mathrm{e}^{-E / R T}+\dot{Q}(r, t) .
$$

These equations are to be solved with the initial conditions

$$
t=0: \quad u=\rho-\rho_{0}=T-T_{0}=p-p_{0}=Y-1=0,
$$

and symmetry conditions at $r=0$. The fluid variables at $r \rightarrow \infty$ for $t \ll t_{\infty}$ are given also by (30). If the activation energy is large, it seems reasonable to expect a negligible initial reaction rate, so that the evolution begins with a first stage of inert heating in which $Y=1$ and the remaining variables are given by the solution of equations (2)-(4) and (29) with $B=0$. Let us denote these frozen, or inert, solutions by $\rho_{\mathrm{f}}, T_{\mathrm{f}}, p_{\mathrm{f}}$ and $u_{\mathrm{f}}$, which obviously depend on the function $\dot{Q}(r, t)$. For the shake of simplicity, we assume that $T_{\mathrm{f}}(r, t)$ has a single maximum.

The chemical reaction comes into play after a time $t_{*}$ such that the instantaneous peak temperature, occurring at $r=0$, attains a cross-over value $T_{*}$, or ignition temperature, that we can define in terms of the physico-chemical parameters of the gas and the duration of the external heating by the relation

$$
t_{\mathrm{e}} \frac{q Y_{0}}{c_{\mathrm{p}} T_{*}} B \mathrm{e}^{-E / R T_{*}}=1,
$$

which, from $T_{\mathrm{f}}\left(0, t_{*}\right)=T_{*}$, determines the ignition time in a first approximation. According to the large value of the activation energy, small changes in temperature, of the order of the Frank-Kamenetskii temperature $R T_{*}^{2} / E$, lead to changes of the order of unity in the reaction rate that, without the effects of the inert temperature, would give rise to the thermal runaway.

On the other hand, due to the finite amount of energy contained in the source, the inert temperature reaches an absolute maximum value $T_{\mathrm{m}}$ at a certain time $t_{\mathrm{m}}$. It becomes apparent 
that if $T_{*}>T_{\mathrm{m}}$ ignition does not occur at times of order $t_{\mathrm{e}}$. If $T_{*}<T_{\mathrm{m}}$, i.e. $t_{*}<t_{\mathrm{m}}$, and the peak temperature, $T_{\mathrm{f}}(0, t)$, grows continuously with time, we can expect an ignition event at times close to $t_{*}$. The transition between subcritical and supercritical regimes corresponds to values of $T_{*}$ close to $T_{\mathrm{m}}$, when the reactive stage, which can end in either thermal runaway or frozen stage, involves values of $T_{\mathrm{f}}-T_{\mathrm{m}}$ of order $R T_{\mathrm{m}}^{2} / E$.

\subsection{Ignition stage and ignition time with a non-instantaneous energy source}

We start the analysis of the ignition with a non-instantaneous source by considering that $T_{*}<T_{\mathrm{m}}$, i.e. those cases for which the peak temperature is still increasing with time when the ignition temperature is reached. According to the definition (31), the effects of the chemical reaction appear at times close to $t_{*}$, but they are confined to a small region, around $r=0$, such that the departures of the temperature from $T_{*}$ are of order $\varepsilon T_{*}$, with $\varepsilon=R T_{*} / E$. Taking into account that, for fixed $t, T_{\mathrm{f}}$ attains a maximum at $r=0$ and assuming that $T_{\mathrm{f}}(0, t)$ is an increasing function of $t$ for $t<t_{\mathrm{m}}$, we can expand $T_{\mathrm{f}}$ around $r=0$ and $t=t_{*}$ as

$$
T_{\mathrm{f}}(r, t)=T_{*}\left[1-\left(\frac{r}{a_{*}}\right)^{2}+\frac{t-t_{*}}{b_{*}}\right]+\mathrm{O}\left(r^{2},\left(t-t_{*}\right)\right),
$$

where the parameters $a_{*}=\mathrm{O}\left(r_{\mathrm{h}}\right)$ and $b_{*}=\mathrm{O}\left(t_{\mathrm{e}}\right)$, characterizing the frozen temperature distribution, are given by

$$
2 T_{*} a_{*}^{-2}=-\frac{\partial^{2} T_{\mathrm{f}}\left(0, t_{*}\right)}{\partial r^{2}}>0, \quad T_{*} b_{*}^{-1}=\frac{\partial T_{\mathrm{f}}\left(0, t_{*}\right)}{\partial t}>0 .
$$

The appropriate time and space variables of order unity to describe the short ignition stage are defined by

$$
\xi=\frac{1}{\sqrt{\varepsilon}} \frac{r}{a_{*}} \quad \text { and } \quad \tau=\frac{1}{\varepsilon} \frac{t-t_{*}}{\left(a_{*} / c_{*}\right)},
$$

where $c_{*}=\sqrt{\gamma R_{\mathrm{g}} T_{*}}$. In terms of these variables, (32) reads

$$
\left(T_{\mathrm{f}}-T_{*}\right) / \varepsilon T_{*}=-\xi^{2}+k_{*} \tau+\mathrm{o}(1),
$$

where $k_{*}=\left(a_{*} / b_{*} c_{*}\right) \sim 1$ is a parameter related to the frozen distribution at $t=t_{*}$.

It is easy to show that, for $\xi$ and $\tau$ of order unity, $p_{\mathrm{f}}-p_{*} \sim \varepsilon p_{*}, \rho_{\mathrm{f}}-\rho_{*} \sim \varepsilon \rho_{*}$, and $u_{\mathrm{f}} \sim \sqrt{\varepsilon} c_{*}$, where $p_{*}=p_{\mathrm{f}}\left(0, t_{*}\right)$ and $\rho_{*}=\rho_{\mathrm{f}}\left(0, t_{*}\right)$. Using the deviations from the frozen distributions,

$$
\begin{array}{ll}
v=\left(u-u_{\mathrm{f}}\right) / \sqrt{\varepsilon} c_{*}, & v=\left(\rho-\rho_{\mathrm{f}}\right) / \varepsilon \rho_{*}, \\
\varphi=\left(T-T_{\mathrm{f}}\right) / \varepsilon T_{*} & \pi=\left(p-p_{\mathrm{f}}\right) / \varepsilon p_{*},
\end{array}
$$

as dependent variables and taking into account the definition (31) of the ignition temperature, equations (2)-(4), (29) and (6) lead, in the limit $\varepsilon \rightarrow 0$, to $Y=1$ and

$$
\begin{aligned}
& \pi=v+\varphi, \\
& \frac{\partial \nu}{\partial \tau}=-\frac{1}{\xi^{j}} \frac{\partial}{\partial \xi}\left(\xi^{j} v\right), \\
& \frac{\partial v}{\partial \tau}=0, \\
& \frac{\partial \varphi}{\partial \tau}=\frac{\gamma-1}{\gamma} \frac{\partial \pi}{\partial \tau}+\frac{k_{*} b_{*}}{t_{\mathrm{e}}} \mathrm{e}^{\varphi+k_{*} \tau-\xi^{2}},
\end{aligned}
$$

whose solution with initial homogeneous conditions at $\tau \rightarrow-\infty$ is found to be $v=v=0$ and $\pi=\varphi=-\ln \left(1-\left(\gamma b_{*} / t_{\mathrm{e}}\right) \mathrm{e}^{k_{*} \tau-\xi^{2}}\right)$, showing the existence of a locus of thermal runaway 
$\tau=k_{*}^{-1}\left(\xi^{2}-\ln \left(\gamma b_{*} / t_{\mathrm{e}}\right)\right)$. The ignition time is the minimum value of $\tau$ at which $\varphi \rightarrow \infty$, so that $\tau_{\mathrm{i}}=-k_{*}{ }^{-1} \ln \left(\gamma b_{*} / t_{\mathrm{e}}\right)$. Therefore, if the frozen temperature, resulting from the external heating, is able to reach the ignition temperature $T_{*}$, we always find an ignition event at a time $t_{\mathrm{i}}=t_{*}-\varepsilon b_{*} \ln \left(\gamma b_{*} / t_{\mathrm{e}}\right)$.

\subsection{Critical conditions for ignition with a non-instantaneous source}

The previous analysis has shown that if $T_{*}<T_{\mathrm{m}}$ and the frozen temperature is an increasing function of time around $t_{*}$, the factor $\mathrm{e}^{k_{*} \tau}$ increases the reaction rate in (40) and removes the criticality of the system. When $T_{*}$ is close to $T_{\mathrm{m}}$, i.e. $t_{*}$ is close to $t_{\mathrm{m}}$, the time $b_{*}=T_{*}\left(\partial T_{\mathrm{f}}\left(0, t_{*}\right) / \partial t\right)^{-1}$ is no longer of order $t_{\mathrm{e}}$, since $\partial T_{\mathrm{f}}\left(0, t_{\mathrm{m}}\right) / \partial t=0$, and the analysis of section 3.1 fails because $k_{*} \rightarrow 0$; the frozen temperature around $T_{\mathrm{m}}$ does not depend linearly on $t$ and, hence, it cannot be represented by (35). This expansion must be replaced by an expansion at $r=0$ and $t=t_{\mathrm{m}}$, which takes into account that $T_{\mathrm{f}}$ attains its maximum value $T_{\mathrm{m}}$.

The analysis of this case proceeds in a way similar to that of section 3.1 . We begin by writing $T_{\mathrm{f}}$ in the form

$$
T_{\mathrm{f}}(r, t)=T_{\mathrm{m}}\left[1-\left(\frac{r}{a_{\mathrm{m}}}\right)^{2}-\left(\frac{t-t_{\mathrm{m}}}{b_{\mathrm{m}}}\right)^{2}\right]+\mathrm{O}\left(r^{2},\left(t-t_{\mathrm{m}}\right)^{2}\right),
$$

where $a_{\mathrm{m}}=\mathrm{O}\left(r_{\mathrm{h}}\right)$ and $b_{\mathrm{m}}=\mathrm{O}\left(t_{\mathrm{e}}\right)$ are defined by

$$
2 T_{\mathrm{m}} a_{\mathrm{m}}^{-2}=-\frac{\partial^{2} T_{\mathrm{f}}\left(0, t_{\mathrm{m}}\right)}{\partial r^{2}}>0, \quad 2 T_{\mathrm{m}} b_{\mathrm{m}}^{-2}=-\frac{\partial^{2} T_{\mathrm{f}}\left(0, t_{\mathrm{m}}\right)}{\partial t^{2}}>0,
$$

since $T_{\mathrm{f}}$ has a maximum at $\left(0, t_{\mathrm{m}}\right)$. The time and space variables are now chosen as

$$
\xi=\frac{1}{\sqrt{\varepsilon}} \frac{r}{a_{\mathrm{m}}} \quad \text { and } \quad \tau=\frac{1}{\sqrt{\varepsilon}} \frac{t-t_{\mathrm{m}}}{\left(a_{\mathrm{m}} / c_{\mathrm{m}}\right)},
$$

where $\varepsilon=R T_{\mathrm{m}} / E$ and $c_{\mathrm{m}}=\sqrt{\gamma R_{\mathrm{g}} T_{\mathrm{m}}}$. The frozen temperature is given by

$$
\left(T_{\mathrm{f}}-T_{\mathrm{m}}\right) / \varepsilon T_{\mathrm{m}}=-\xi^{2}-k_{\mathrm{m}}^{2} \tau^{2}+\mathrm{o}(1),
$$

where $k_{\mathrm{m}}=a_{\mathrm{m}} / b_{\mathrm{m}} c_{\mathrm{m}} \sim 1$. For the sake of simplicity, we limit ourselves to cases in which $\dot{Q}(r, t)$ is such that $\partial^{2} T_{\mathrm{f}}\left(0, t_{\mathrm{m}}\right) / \partial r \partial t=0$.

We use the deviations from the frozen distributions,

$$
\begin{array}{ll}
v=\left(u-u_{\mathrm{f}}\right) / \varepsilon c_{\mathrm{m}}, & v=\left(\rho-\rho_{\mathrm{f}}\right) / \varepsilon \rho_{\mathrm{m}}, \\
\varphi=\left(T-T_{\mathrm{f}}\right) / \varepsilon T_{\mathrm{m}} & \pi=\left(p-p_{\mathrm{f}}\right) / \varepsilon p_{\mathrm{m}},
\end{array}
$$

as dependent variables, where $u_{\mathrm{f}} \sim \sqrt{\varepsilon} c_{\mathrm{m}}$ and, although $\left(T_{\mathrm{f}}-T_{\mathrm{m}}\right) / T_{\mathrm{m}} \sim \varepsilon,\left(p_{\mathrm{f}}-p_{\mathrm{m}}\right) / p_{\mathrm{m}}$ and $\left(\rho_{\mathrm{f}}-\rho_{\mathrm{m}}\right) / \rho_{\mathrm{m}}$ are, in general, of order $\sqrt{\varepsilon}$ because $\partial p_{\mathrm{f}}\left(0, t_{\mathrm{m}}\right) / \partial t$ and $\partial \rho_{\mathrm{f}}\left(0, t_{\mathrm{m}}\right) / \partial t$ can be different from zero.

In the limit $\varepsilon \rightarrow 0$ we obtain, from equations (2)-(4), (29) and (6) that $Y=1$ and, after using the equation of state $\pi=v+\varphi$ to eliminate $v$, the following system of equations for $v$, $\varphi$ and $\pi$ :

$$
\begin{aligned}
& \frac{\partial v}{\partial \tau}+\frac{1}{\gamma} \frac{\partial \pi}{\partial \xi}=0 \\
& \frac{\partial \varphi}{\partial \tau}-\frac{\gamma-1}{\gamma} \frac{\partial \pi}{\partial \tau}=\delta_{\mathrm{m}} \exp \left(\varphi-\xi^{2}-k_{\mathrm{m}}^{2} \tau^{2}\right), \\
& \frac{1}{\gamma} \frac{\partial \pi}{\partial \tau}+\frac{1}{\xi^{j}} \frac{\partial\left(\xi^{j} v\right)}{\partial \xi}=\delta_{\mathrm{m}} \exp \left(\varphi-\xi^{2}-k_{\mathrm{m}}^{2} \tau^{2}\right)
\end{aligned}
$$




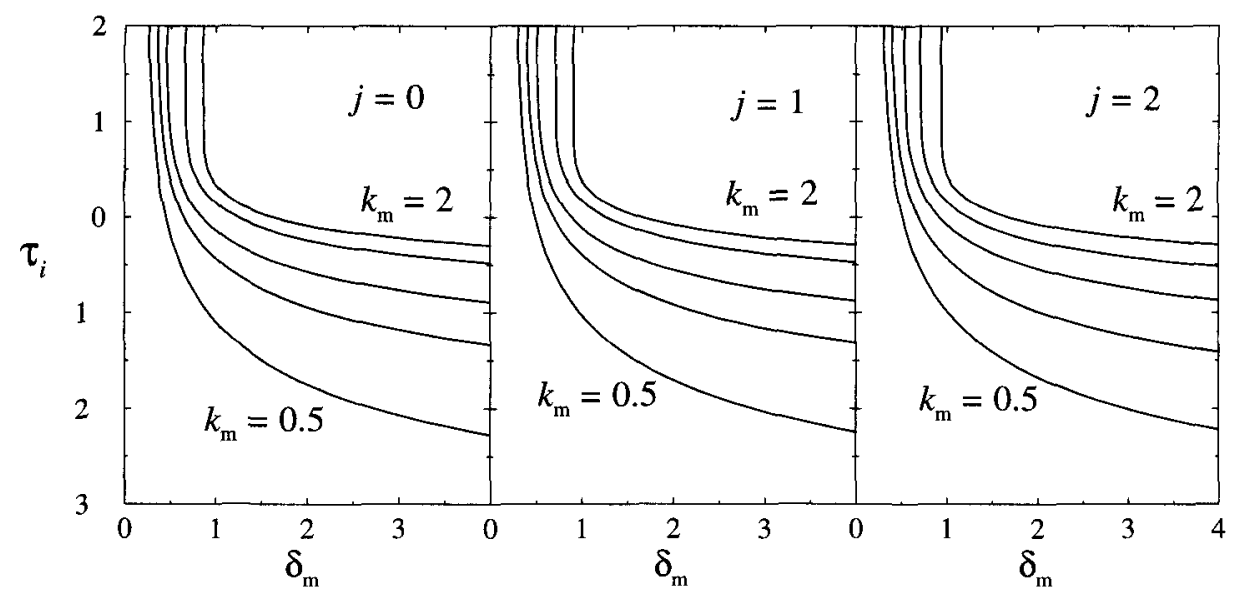

Figure 3. The ignition time $\tau_{\mathrm{i}}$ as a function of $\delta_{\mathrm{m}}$ for $k_{\mathrm{m}}=0.5,0.75,1,1.5$ and 2 .

This must solved with symmetry conditions at $\xi=0$ and homogeneous initial conditions $v=\varphi=\pi=0$ at $\tau \rightarrow-\infty$, holding also for $\xi \rightarrow \infty$. The Damköhler number, $\delta_{m}$, is defined by

$$
\delta_{\mathrm{m}}=\frac{a_{\mathrm{m}}}{c_{\mathrm{m}}} \frac{q Y_{0}}{c_{\mathrm{p}} T_{\mathrm{m}}} \sqrt{\frac{E}{R T_{\mathrm{m}}}} B \exp \left(\frac{-E}{R T_{\mathrm{m}}}\right),
$$

of order unity for near-critical ignition sources.

The solution of equations (46)-(48) leads to a thermal runaway, or blow-up, at $\xi=0$ and $\tau=\tau_{\mathrm{i}}\left(\gamma, k_{\mathrm{m}}, \delta_{\mathrm{m}}\right)$ for values of $\delta_{\mathrm{m}}$ larger than the critical value $\delta_{\mathrm{m}, \mathrm{c}}\left(\gamma, k_{\mathrm{m}}\right)$. For lower values of $\delta_{\mathrm{m}}$, ignition fails to occur at times of order $t_{\mathrm{e}}$, and $v$ and $\pi$ decay to zero for large values of $\tau$, while $\varphi$ reaches an asymptotic distribution as a result of the heat released by the chemical reaction. These equations are solved, using the same method as in section 2 , for $\gamma=1.4$ and several values of $k_{\mathrm{m}}$ to obtain the critical values of the Damköhler number and the ignition time for each supercritical value. Figure 3 shows the ignition time as a function of $\delta_{\mathrm{m}}$ for different values of $k_{\mathrm{m}}$. A similar analysis of critical conditions for a different ignition problem is given in [10].

\section{Concluding remarks}

We have analysed, using large-activation-energy asymptotics, the evolution of a gaseous reacting mixture after the deposition of a finite amount of energy in a small region of gas. In order to identify the relevant physical mechanisms, we have defined the following characteristic times:

(a) $t_{\mathrm{e}}$, the characteristic time of energy deposition;

(b) $t_{\mathrm{a}}$ and $t_{\mathrm{c}}$, times associated to expansion waves and heat conduction, respectively, in the region where the temperature differs from its maximum value by an amount of the order of the Frank-Kamenetskii temperature;

(c) $t_{\mathrm{ch}}$, the chemical time evaluated at the maximum temperature just after the energy addition.

When these times satisfy $t_{\mathrm{e}} \ll t_{\mathrm{ch}} \sim t_{\mathrm{a}} \ll t_{\mathrm{c}}$, we can neglect heat conduction effects and consider that the source releases the energy instantaneously at constant volume. After 
the deposition of energy, the high temperature ${ }^{\prime}$ rggers the chemical reaction and the gradients associated with the overpressures generated du ring the external heating stage lead to expansion waves that cool the hot-spot. Hence, the evolution is determined by the competition between both effects. The ratio $t_{\mathrm{a}} / t_{\mathrm{ch}}$ defines the Damköhler number, $\delta$, which turns out to be the main parameter in the problem. We have shown that, for each symmetrical configuration, there exists a critical value of order unity, $\delta_{\mathrm{c}}$, which depends only on $\gamma$, such that ignition is achieved only for $\delta>\delta_{\mathrm{c}}$ at an ignition time $\tau_{\mathrm{i}}(\delta, \gamma)$ given in figure 2. The solution of (25)-(27) for $\tau_{\mathrm{i}}-\tau \ll 1$ shows that both temperature and pressure at the centre of the hot-spot become unbounded when $\tau=\tau_{\mathrm{i}}$, but not in the same way as shown in [3]. For very large values of $\delta$, the chemical time is so small that the expansion does not play a role during the ignition stage. This case corresponds to a constant-volume thermal runaway with $t_{\mathrm{i}}=t_{\mathrm{ch}}$.

Notice that the criticality is a consequence of the expansion cooling. In other works, e.g. $[3,5]$, this effect does not appear, and the results show the existence of a thermal runaway for any value of the parameter measuring the ratio of the acoustic time to the chemical time, i.e. the container size in [3] and the non-dimensional temperature gradient in [5].

When the energy source is not instantaneous, i.e. $t_{\mathrm{e}} \sim t_{\mathrm{a}}$, we find two temporal stages: a first inert heating stage of a duration defined by the time required to attain a cross-over temperature, which determines the ignition time in the first approximation, and a shorter reactive stage ending in a thermal runaway; the analysis of this stage allows us to obtain a small correction in the ignition time.

It should be noted that the ignition of the mixture does not guarantee the formation of a travelling combustion front. Thus, the conditions for ignition must be seen as necessary, but not sufficient, to initiate a detonation. The direct initiation problem, i.e. the detonation resulting from the deposition of a sufficient amount of energy, was first addressed by Zel'dovich [11] and more recently by $\mathrm{He}$ and Clavin [12], who obtained a value of the minimum energy to be released by an instantaneous point source in order to develop a detonation wave. The unsteady effects, neglected in the model proposed by He and Clavin, has been incorporated by Eckett et al [13]. A different approach to this problem is given by Short [14]. He considers a reactive mixture subject to order $\varepsilon$ disturbances. Although this assumption constitutes a serious restriction, when used in conjunction with the limit $\delta \gg 1$ it allows him to obtain analytically the critical conditions leading to a ZND detonation. 